Int. J. Dev. Biol. 61: 319-327 (2017)

doi: 10.1387/ijdb.160250ao

\title{
The SUMO-targeted ubiquitin ligase, Dgrn, is essential for Drosophila innate immunity
}

\author{
BELLA KOLTUN', ELIZA SHACKELFORD, FRANÇOIS BONNAY², NICOLAS MATT², \\ JEAN MARC REICHHART ${ }^{2}$ and AMIR ORIAN*,1 \\ ${ }^{1}$ Rappaport Research Institute and Rappaport Faculty of Medicine, Technion Integrated Cancer Center, Technion \\ - Israel Institute of Technology, Haifa, Israel and 2 UPR9022 du CNRS, Institut de Biologie Moléculaire et Cellulaire \\ Université de Strasbourg, France
}

\begin{abstract}
The ability of metazoans to combat pathogenic infection involves both systemic and local responses to the invading pathogens. Ubiquitin and SUMO pathways molecularly regulate the response to infection, immune signaling and gene expression. Here, we report that Degringolade (Dgrn, CG10981), a SUMO-targeted ubiquitin ligase connecting the two pathways, is essential for the innate immunity response in Drosophila. dgrn $^{D K}$ null and heterozygous mutant adult flies are severely immune-compromised and succumb rapidly to both pathogenic bacteria and fungi infections. The sensitivity to infection stems from the inability to produce multiple anti-microbial peptides, and transcriptional analyses suggest that the overexpression of Dgrn enhances the transcriptional output of the NF- $\mathrm{B}$ related Toll and immune deficiency (IMD)-pathways. Moreover, expression of Dgrn alleviated the inhibitory impact of the cytoplasmic NF- $\kappa$ B inhibitor Cactus and the nuclear corepressor Groucho/TLE (Gro). Additionally, we found that Dgrn is required for the local regenerative response of the mid-gut following infection. Upon oral infection, dgrn mutant flies fail to activate the Delta-Notch pathway in stem cells and enteroblasts, and are unable to regenerate and replace the damaged and dying enterocytes. Interestingly, the ubiquitin-specific protease CG8334 (dUSP32) dUSP11) antagonizes Dgrn activity in the gut, and halving the dose of CG8334 restores Delta-Notch signaling and rescues the lethality observed in dgrn mutants. Collectively, our data suggest that Dgrn is essential for both systemic and local tissue response to infection.
\end{abstract}

KEY WORDS: Drosophila, innate-immunity, STUbL, SUMO, ubiquitin

\section{Introduction}

Post-transcriptional modifications (PTMs) by ubiquitin (Ub) and ubiquitin-like proteins (UbLs) regulate both innate and adaptive immune responses (van der Veen et al., 2012; Everret et al., 2013; Popovich et al., 2014; Heaton et al., 2016). In mammals and Drosophila, NF-кB pathway and REL-transcription-factors (TFs) form the core network that regulates the host response against infection. REL TFs are essential for development and their deregulation contributes to a wide variety of human diseases (Ghosh et al., 2012). The Drosophila NF-кB protein family is comprised of three REL TFs: Dorsal, Dif and Relish. Specifically, in adult flies, activation of Toll pathway genes is mediated by TF Dif, in response to Gram-positive bacteria and fungal infections. On the other hand, the immune deficiency (IMD) pathway and TF Relish are required to cope with Gram-negative bacteria (Buchon et al., 2014). In un-stimulated cells, REL TFs are sequestered in the cytoplasm by $\mathrm{I}_{\mathrm{K}} \mathrm{B} / \mathrm{Cactus}$ inhibitory proteins. Upon a variety of signals (e.g., infection, cytokines, etc.), receptor-mediated activation initiates a signaling cascade involving multiple events of ubiquitylation, leading to the degradation of the inhibitory proteins (Kanarek et al., 2012). Subsequently, REL proteins translocate to the nucleus where they regulate the expression of target genes (Wan et al., 2010). In Drosophila exposure to microbial threats at the level of epithelia drives a potent local immune response based on anti-microbial peptides

Abbreviations used in this paper: AMP, anti-microbial peptide; Dgrn, degringolade; DSS, dextran sulfate sodium; Gro, groucho; IMD, immune deficiency; PTM, post-transcriptional modification; SUMO, small ubiquitin-like modifier; UbL, ubiquitin-like protein.

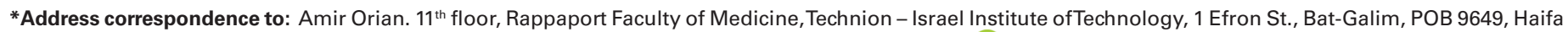
31096, Israel. e-mail: mdoryan@techunix.technion.ac.il -Tel.: 972-4-829-5448/9. Fax: 972-4-852-394. (iD http://orcid.org/0000-0002-8521-1661
}

Supplementary Material (one figure) for this paper is available at: http://dx.doi.org/10.1387/ijdb.160250ao

Accepted: 10 August, 2016.

ISSN: Online 1696-3547, Print 0214-6282 
(AMPs) synthesis and activation of resilience mechanisms such as enterocyte-renewal in the gut epithelium (Ferrnadon D, 2013). In addition, invasion of the body-cavity by microbes activates the systemic innate immune response, which consist in the massive release of AMPs by the fat-body (the equivalent of the mammalian liver) (Ferrandon et al., 2007).

Similar to ubiquitylation, UbL pathways regulate innate response, and one such UbL pathway is SUMOylation. SUMOylation is initiated by the activation of SUMO, a ubiquitin-like molecule, by the SUMO-specific activating enzyme (E1). It also requires the activity of the SUMO conjugating E2 (Ubc9) and E3-SUMO ligases. SUMOylation is reversible by the activity of SUMO-specific peptidases SENPs (Flotho et al., 2013). SUMOylation regulates $\mathrm{NF}-\kappa \mathrm{B}$ signaling and NF- $\mathrm{BB}$-dependent transcription in the nucleus. It also regulates the activity of other immune pathways involved in the response to infection (Liu 1998; Lee et al., 2011; Lee 2009; Decque et al., 2016). Indeed, mutants deficient for the SUMO pathway exhibit an abnormal immune response, and targeted inactivation of the SUMO-conjugating E2, Ubc9, was developed by Listeria to evade recognition by the immune system and promote efficient infection (Ribet et al., 2010; Paddibhatla et al., 2010).

Ubiquitylation and SUMOylation are connected molecularly in part by SUMO-targeted ubiquitin ligases (STUbL). Conserved from flies to humans, STUbLs are a unique group of RING proteins: they bind non-covalently to the SUMO moiety of SUMOylated proteins via their N-terminal SUMO Interacting Motifs (SIMs) domains, and subsequently target the SUMOylated protein for ubiquitylation via their C-terminal RING domain. Therefore, they are able to "sense" SUMOylated proteins and modify them by ubiquitylation. STUbL-mediated ubiquitylation in many cases leads to proteasomal degradation, but also affects protein-protein interactions, protein localization and activity (Sriramachandran and Dohmen. 2014).

In the fly, a single STUbL exists termed Degringolade (dgrn, CG10981), which is essential for embryonic development (Abed et al., 2011; Berry et al., 2011). Dgrn is required for proper response to DNA damage/replication stress, and the regulation of gene expression during segmentation and sex determination (Ryu et al., 2015; Abed et al., 2011; Barry et al., 2011). We previously found that $d g r n^{D K}$ null adult males are fertile, yet $d g r n^{D K}$ null females are viable however sterile, laying embryos that do not hatch (Barry et al., 2011). Termed the maternal effect female sterile, this phenotype is also observed in mutants of the Toll pathway that are characterized by a defective immune system (Anderson et al., 1985).

Here, we report that Dgrn is a new key ubiquitin ligase required for both systemic and local immunity in Drosophila. First, dgrnadult flies are immune-compromised and sensitive to multiple pathogenic threats. We found that Dgrn is essential during systemic immune response to infection essential for the transcription of Toll- and IMD-dependent gene expression. Second, Dgrn was required for a Notch-dependent, local stem cells regeneration upon gut infection. We also identified CG8334, an ubiquitin hydrolase, as a gene that antagonizes dgrn function in the gut.

\section{Results}

\section{$\mathrm{dgrn}^{\mathrm{DK}}$ null adults are immuno-compromised and hyper-sensitive to infection}

In the adult fly, the Toll pathway is required for coping with Gram-positive bacteria and fungal infections (Buchon et al., 2014). Therefore, we first tested the ability of $d g r n^{D K}$ null mutants to resist
A

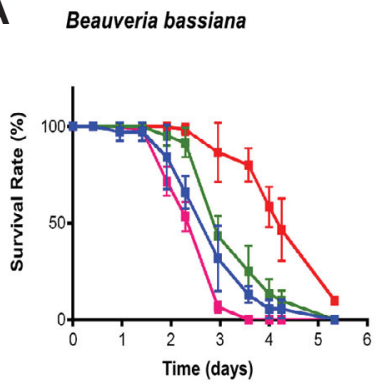

$\mathbf{A}^{\prime}$

Beauveria bassiana

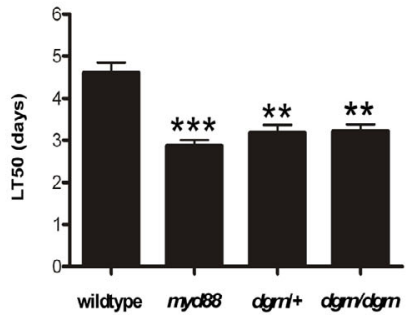

B

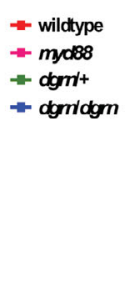

$B^{\prime}$

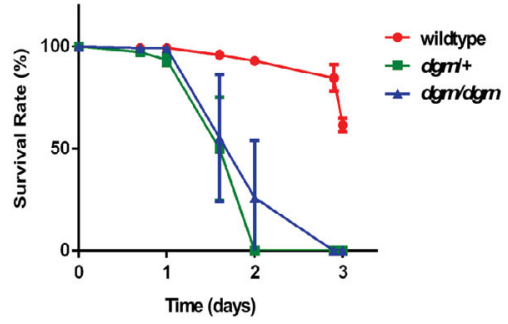

Serratia marcescens (Db 11)

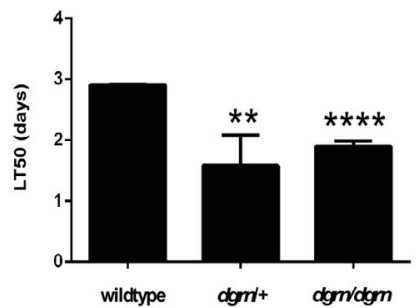

C Dextran Sulfate Sodium (DSS) $5 \%$

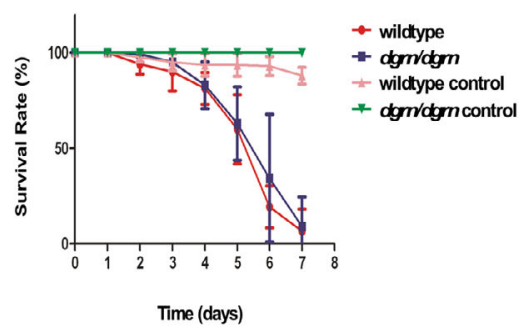

$C^{\prime}$

Dextran Sulfate Sodium (DSS) $5 \%$

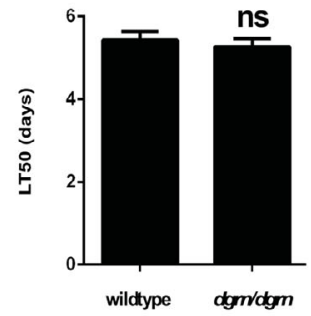

Fig. 1. dgrn $^{D K}$ mutant flies are immune-compromised and sensitive to infection by diverse pathogenic microorganisms. (A-C) Survival analysis of adult flies upon infection with the indicated fungus, bacteria or challenges by Dextran Sulfate Sodium (DSS) was performed as described under Materials and Methods. (A, $\left.\mathbf{A}^{\prime}\right)$. Infection with Beauveria bassiana fungal spores, myd88c ${ }^{03881}$ serves as a positive reference. (B, $\left.\mathbf{B}^{\prime}\right)$ Oral infection with the $\mathrm{Gram}^{-1}$ bacteria Serratia maracescans (Sm, Db11). (C, $\mathbf{C}^{\prime}$ ) Exposure to 5\% DSS in $A^{\prime}-C^{\prime}$ LT50 is determined as the time in days when $50 \%$ of the infected flies succumbed to infection. For pathogenic challenges $n=20$ for each genotype, and $n=50$ in DSS exposure in each individual experiment. Three independent biological repeats were performed in each set of experiments. 
fungal infection by performing natural Beauveria bassiana infection, a classical experiment to assess the Toll-pathway (Lemaitre et al., 1997). We observed that under non-challenged conditions, the survival of adult dgrn mutants was identical to that of wild-type flies. However, exposure to Beauveria bassiana spores resulted in earlier lethality of $d g r n^{D K}$ heterozygous and homozygous adults to the fungus compared to wild-type flies. This sensitivity was similar to that observed with mutants for Myd88, an essential Toll pathway scaffold protein (Fig. 1A,A', data not shown).

The enhanced susceptibility of $d g r n^{D K}$ mutants to infection was also observed upon exposure to Gram-negative bacteria, known agonists of the IMD pathway. dgrn ${ }^{D K}$ mutant flies exhibit enhanced sensitivity to intestinal infection with the Gram-negative bacteria Serratia marcescens (Sm DB11) and Pseudomonas aeruginosa (PA14; Fig. $1 \mathrm{~B}, \mathrm{~B}$ ', $\mathrm{S} 1 \mathrm{~A})$. This sensitivity was similar to that observed in kenny ${ }^{1}$ mutants, deficient for a central kinase within the IMD pathway (not shown). Nonetheless, the survival of $d g r n^{D K}$ null mutants that were exposed to a chemical intestinal stressor ( $5 \%$ Dextran Sulfate Sodium, DSS) was identical to that of wild-type flies (Fig. $1 \mathrm{C}, \mathrm{C}^{\prime}$ ), ruling out the possibility that the observed dgrn susceptibility to pathogenic infections originated from an epithelial weakness.

\section{dgrn ${ }^{\mathrm{DK}}$ null adults fail to express AMP genes upon infection}

A hallmark of the systemic immune response is the production of anti-microbial peptides (AMPs, Ferrandon et al., 2007). We hypothesized that the increased sensitivity to infection observed in $d g r n^{D K} \mathrm{mu}$ tants stems from the inability to express AMP genes. Therefore, we monitored the expression of endogenous mRNA of AMP genes upon various pathogenic challenges. In contrast to wild-type flies, $d g r n^{D K}$ mutants failed to express Toll-related AMPs (drosomycin, IM-1, metchnikowinand defensin) in response to infections with the Gram-positive bacteria M. luteus or the fungus Beauveria Bassiana (Figs. $2 \mathrm{~A}-\mathrm{F}$ ). These decreased AMPs mRNA levels were comparable to those of myd88 mutant flies. Likewise, we observed that Dgrn was essential for the expression of the IMD-dependent AMP genes attacin- $A,-B,-C,-D$, diptericin and drosocin upon infection with the Gram-negative

Fig. 2. dgrn is required for the expression of Toll- and immune deficiency (IMD)-dependent anti-microbial peptides (AMPs) upon infection. Real-time qPCR analyses of mRNA levels of the indicated AMPs. Adult flies with the indicated genotypes were infected with $\mathrm{B}$. bassiana spores (A-C), pricked with PBS (control), or either with $\mathrm{Gram}^{(+)}$bacteria M. luteus, (D-F), or the Gram ${ }^{-1}$ bacteria E. coli. (G-L) AMP tested: drosomycin (A, D), IM-1 (B), metchnikowin (C, $F)$, defencin $(E)$, attacinA (G), attacinB $(H)$, attacinC (I), attacinD (J), diptericin (K), drosocin (L). myd88 c038 81 is a mutant within the Toll pathway (A-F). keyc0283' is a mutant of the obligatory kinase kenny within the IMD pathway (G-L). $n=10$ for each genotype, and three biological repeats for each set of experiments were performed.

bacteria $E$. coli. Similar decreased AMPs levels were observed in IMD-deficient kenny mutant flies (Fig. 2 G-L). Altogether, these results indicate that $d g r n$ is required for Drosophila innate immune response. dgrn mutants are immune-compromised and fail to express AMP genes in response to infection with pathogens that activate either the Toll or IMD signaling pathways.

\section{Dgrn enhances NFKB-dependent gene expression in S2 Drosophila cells}

To gain insight into the role of dgrn in the transcriptional activation of AMP genes, we used NF-KB luciferase reporter assays in $\mathrm{S} 2$ cells. We activated the IMD or Toll pathway by over-expressing the constitutively active IMD pathway PGRP-LCa receptor or the constitutively active truncated Toll receptor (Toll${ }^{\Delta L R R}$ ), respectively (Goto et al., 2008) (Fig. 3A). Co-expression of Dgrn enhanced the transcriptional activation of the $\mathrm{kB}$ reporter by 2-3 fold compared to constitutively active receptors alone (Fig. 3A). Next, we studied the transcriptional effect(s) of Dgrn on the activation of the Toll pathway and its TF Dif. In unchallenged cells, Dif is sequestered predominantly in the cytoplasm by the $1 \mathrm{kB} \alpha$-like inhibitor, Cactus. Toll-induced phosphorylation of Cactus results in the ubiquitylation and degradation of Cactus, enabling the nuclear translocation of Dif. In the nucleus, Dif induces the transcription of AMP genes such as drosomycin (Meng et al., 1999). As expected, ectopic expression of Cactus inhibited a Toll-mediated activation of

A

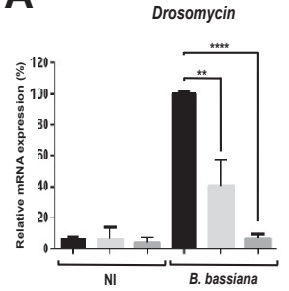

D Drosomycin

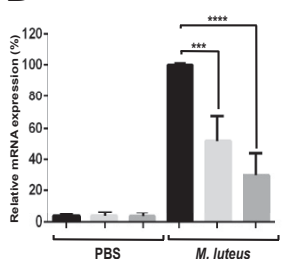

G

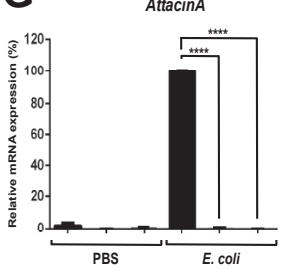

$\mathbf{J}$

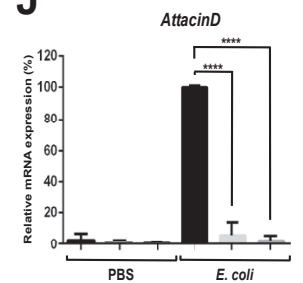

B

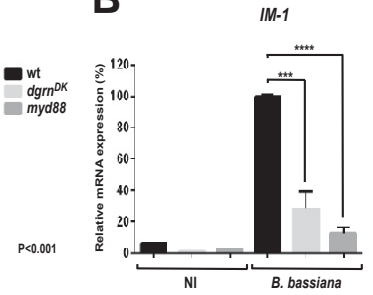

E
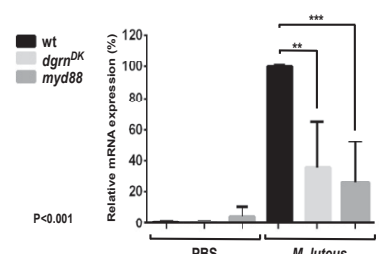

H
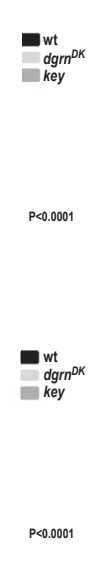

K

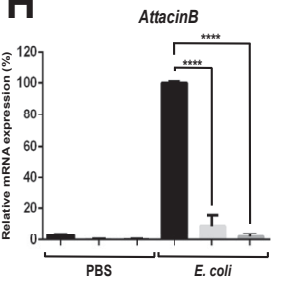

$\mathrm{K}$

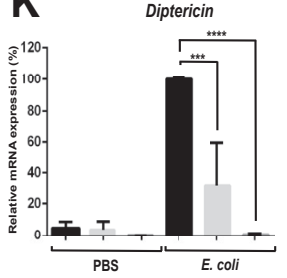

C
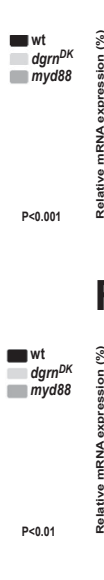

$F$
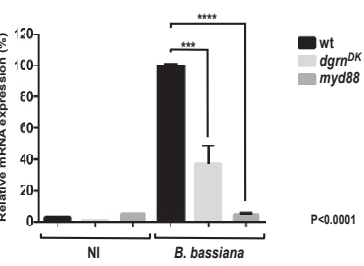

$P<0.0001$
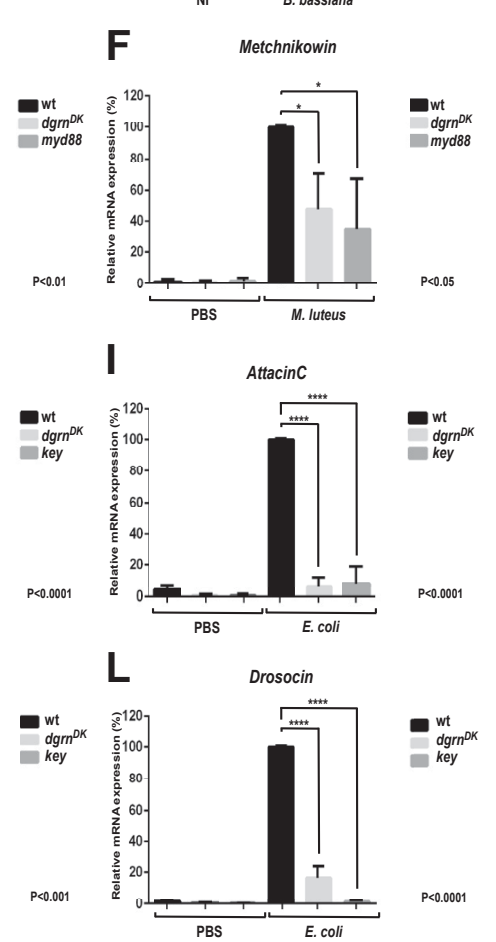
drosomycin-Luc (Drs-Luc; Fig. 3B). However, co-expressing Dgrn alleviated Cactus inhibition and fully restored luciferase expression. Importantly, this function of Dgrn required its ligase activity as a catalytically inactive mutant in the RING domain (Dgrn ${ }^{\mathrm{HC} / \mathrm{AA}}$ ) was unable to alleviate Cactus-mediated repression (Fig. 3B). These results suggest that Dgrn functions in parallel or downstream of Cactus in the Toll pathway signaling cascade.

One potential downstream nuclear substrate of Dgrn in this process would be the co-repressor Groucho that, during development Gro binds to REL transcription factor Dorsal (Cinnamon et al., 2008). Moreover, we previously described that Dgrn inactivates the SUMOylated co-repressor Gro during development (Abed et al., 2011). Indeed, we observed that Gro expression inhibited the Tollmediated activation of the Drs-Luc reporter (Fig. 3C). Remarkably, the co-expression of Dgrn, but not its catalytically inactive mutant, alleviated Gro-dependent repression (Fig. 3C).

Next, we tested whether Dgrn's potentiation of Drs transcriptional activity in this setting required the TF Dif. Suppression of Dif via RNAi, but not control GFPi, resulted in reduced Dif protein levels and a concomitant decline in Toll ${ }^{\triangle \mathrm{LRR}}$-dependent transcriptional activation (Figs. 3D, D'). Remarkably, over-expression of Dgrn in these conditions restored Toll ${ }^{\triangle L R R}$-dependent activation (Figs. 3D). Furthermore, the RNAi-dependent reduction in Dif protein levels were restored by the expression of Dgrn (Fig. 3D'). Taken together these results suggest that the residual Dif protein observed in Dgrn expressing cells are more potent in activating transcription, or that Dgrn functions in a Dif-independent parallel pathway. Collectively, this set of gain of function experiments suggests that Dgrn potentiates Toll- and IMD-dependent transcriptional activation and likely antagonizes the co-repressor Gro at the level of Toll pathway target genes.

\section{Dgrn is required for local regenerative response of the gut to infection}

In addition to the systemic response to infection, the local tissue response to infection is central for combating pathogens invasion (Lemaitre et al., 2007). In both vertebrates and flies, the gut is the habitat of commensal bacteria but also the entry gate for pathogenic micro-organisms. Upon infection, enterocytes are lost and the integrity of the epithelial tissue is hampered. Subsequently, a regenerative response is initiated by signals secreted by the dying enterocytes, and results in hyper-proliferation of intestinal stem cells (ISCs) that are required to replace the damaged/dying enterocytes (ECs). One signaling pathway that is involved in regeneration in response to infection is Notch. In this case, infection of the gut epithelia results in enhanced expression of the Notch ligand Delta receptor on the surface of intestinal stem-cells (ISC) and the subsequent activation of Notch signaling and expression of Notch target genes in the adjacent cells, the enteroblasts (Jianian et al., 2012).

Since $d g r n^{D K}$ mutants are sensitive to oral infection with $S m D b 11$ (Fig. 1B), we monitored the local changes and activity of Notch signaling in the adult mid-gut in mock or Sm challenged flies. Upon infection, control guts showed an increase in the expression of the Notch ligand, Delta, on the surface of ISC and hyper-activation of the Notch-reporter transgene in enteroblasts (EBs) (Figs. 4 A-B'). These changes were also accompanied by an increase in mitosis (cells positive for the mitotic marker p-histone-3) (Figs. 4 A",B"). In sharp contrast, dgrn mutants failed to enhance delta expression on the surface of ISC and showed only minimal expression of the
A

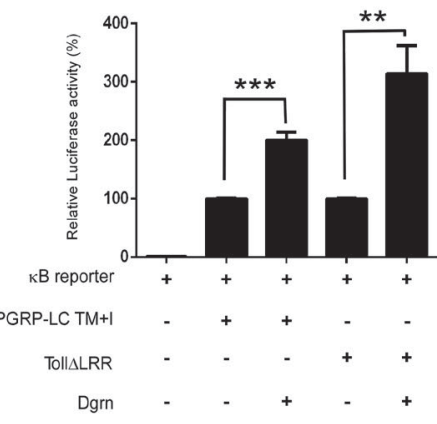

D

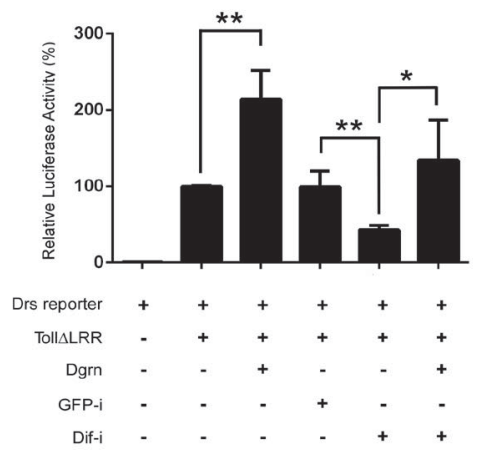

B

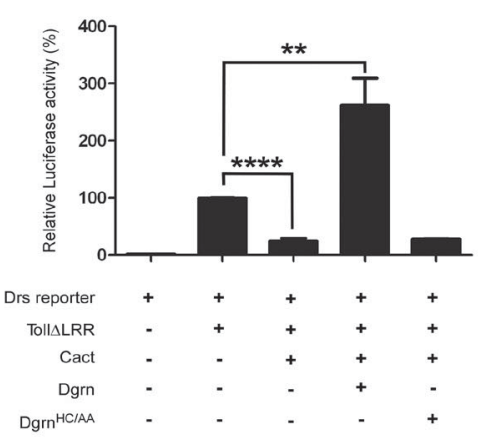

$\mathbf{E}$

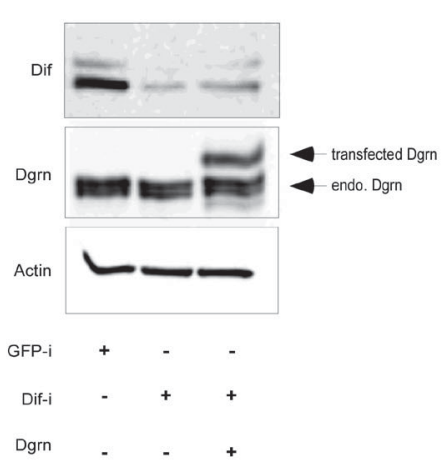

C

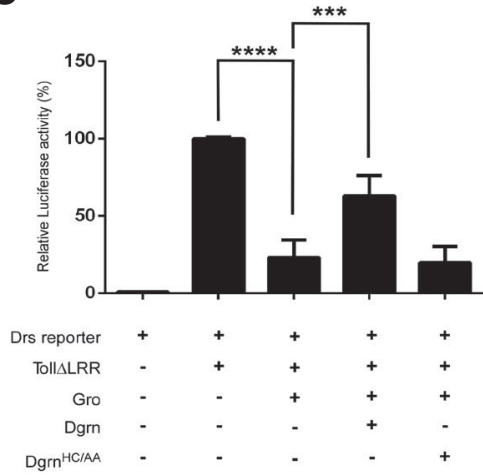

Fig. 3. Dgrn enhances Toll- and IMD-dependent transcriptional activation in S2 Drosophila cells. (A-D) Relative $N F \kappa B$-luciferase reporter activity in S2 cells transfected with the indicated plasmids and luciferase reporters as described under Materials and Methods. (A) Expression of Dgrn enhances the Toll- or IMD-dependent activation of a canonical NFKB reporter. (B) Tol/LLRR-dependent activation of the Toll-specific Dorsomycin (Drs)-Luciferase reporter is suppressed by co-expressing Cactus. Co-expression of Dgrn, but not Dgrn RING mutant (Dgrn $\left.{ }^{H C / A A}\right)$, alleviates Cactus inhibition. (C) Activation of the Dorsomycin (Drs)-Luciferase is suppressed by co-expressing Gro. Co-expression of Dgrn, but not Dgrn ${ }^{H C / A A}$, alleviates Gro-dependent repression. (D) Dgrn enhancing Toll-mediated activation is reduced in S2 cells where Dif is targeted by RNAi. (E) Western blotanalysis of the protein levels of Dif and Dgrn in RNAi treated cells used in (D). GFP-i serves as a non-specific RNA-i control. Actin serves as a loading control. In all experiments, three independent biological repeats were performed. 

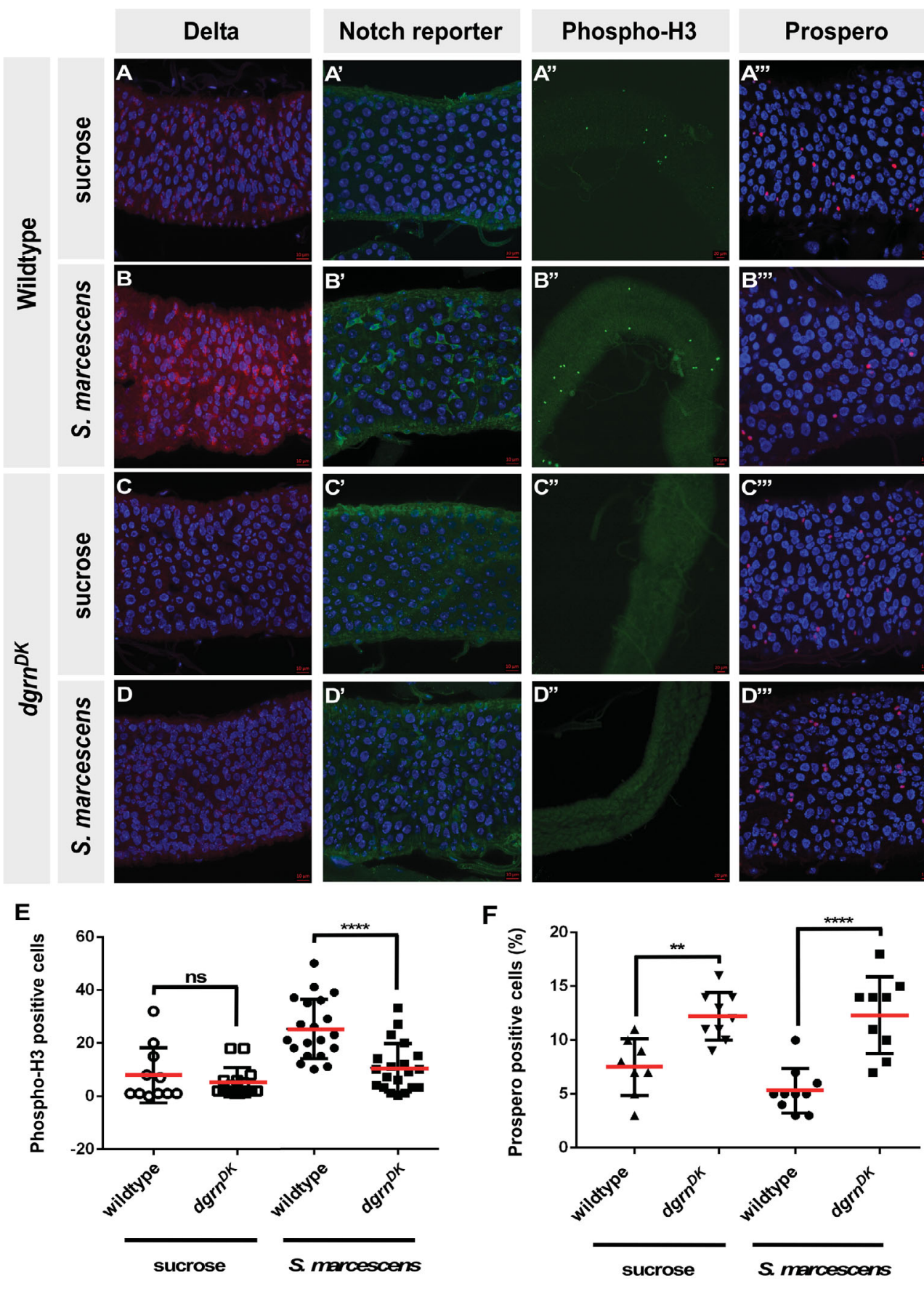

Fig. 4. Dgrn is required for local gut response to oral infection with S. marcescens. (A-D"') Representative confocal images of Drosophila adult mid-gut of the indicated genotypes wild-type (A-B"') and dgrnDK $\left(C-D^{\prime \prime \prime}\right)$. (A-A', $\left.C-C^{\prime \prime \prime}\right)$ are guts derived from sucrose-fed adult flies. (B- $\left.B^{\prime \prime \prime}, D-D\right)$ are guts derived from flies orally infected with $\mathrm{Sm}(\mathrm{Db} 11)$. Forty-eight hours after infection, guts were analyzed for the expression of the Notch ligand and ISC marker, Delta (Red, A-D) and Notch reporter activity (Green, Gbe-Su(H)>lacZ; $\left.A^{\prime}-D^{\prime}\right)$. Mitosis was determined by phospho-Histone-3 (Green, Phsopho-H3; $A^{\prime \prime}-D^{\prime \prime}$ ) and $\alpha$-Prospero antibody (Red) marks entero-endocrine cells ( $\left.A^{\prime \prime \prime}-D^{\prime \prime \prime}\right)$. DAPI (Blue) marks DNA. (E) Quantification of mitotic cells (p-H3 positive) in wild-type and dgrnDK guts following oral infection with $\mathrm{Sm}$ or sucrose control. Each dot represents the number of phsopho-Histone-3 positive cells in whole gut. In all experiments at least 7 individual guts were examined for each genotype, and three biological independent repeats were performed. (F) Quantification of entero-endocrine cells (Prospero ${ }^{(+)}$) in wild-type and dgrnDK guts following oral infection with Sm(Db11) or sucrose control. Individual guts are represented as a single dot on the graph. The number of positive cells per gut section was normalized to total number of cells observed in the same section.
Notch-reporter in EBs (Figs. 4 C-D'). Likewise, dgrn mutants showed overall less mitosis in comparison to wild-type derived guts (compare Fig. 4 A" and 4 B",C",D", and quantitated in $4 \mathrm{E})$. In addition, we evaluated the number of entero-endocrine cells (EEs, Prospero positive cells) in control or Sm-infected guts. We found that the number of EEs was higher in dgrn mutants than in wild-type flies even without infection. However, we did not observe a dramatic change in the average number of EEs upon infection(Figs. 4A"',B"',C"',D"',4F). We concluded that Dgrn is required for the Notch-dependent-regenerative response in the gut upon infection.

\section{The iso-peptidase CG8334 antago- nizes Dgrn during the local gut response to infection}

Ubiquitylation is reversible by the activity of ubiquitin-specific proteases (Heride et al., 2014). In this regard, of interest is CG8334, which is closely related to both dUSP11 and dUSP32. USP11 is a known component of the DNA damage response ( $\mathrm{Yu}$ et al., 2016) and was recently identified as an RNF4-associated protein capable of counteracting RNF4 function in human cancer cells (Hendriks et al., 2015). Therefore, we hypothesized that the fly ortholog CG8334 could potentially antagonize Dgrn function in vivo. Performing a genetic epistasis assay, we found that while dgrn heterozygous mutant flies (+;+;dgrn $\left.{ }^{D K} /+\right)$ rapidly succumb to $S m$ infection, this lethality was greatly suppressed by halving the dose of CG8334 (+

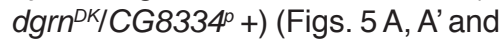
$\mathrm{S} 1 \mathrm{~B})$. Moreover, halving the dose of CG8334 (+ dgrn ${ }^{D K} / \mathrm{CG} 8334^{\circ}+$ ) restored the ability of ISCs to express Delta in the absence and upon Sminfection (Figs. 5B-D'" and quantitated in $5 E$ ). These data fit well with the observation that in human gliomaderived cells USP11 expression is repressed by Notch (Wi et al., 2014), implying that CG8334 is a negative regulator of Notch signaling and Dgrn during the local response to infection and gut homeostasis. Yet, halving the dose of CG8334 did not restore the inability of dgrn mutants to express the anti-microbial peptide att upon 
pricking with E. coli (not shown). Suggesting that the activity of CG8334 is context specific, antagonizing Dgrn activity in the gut in the context of Notch signaling, but not in the context of the IMD pathway and the transcription of AMPs upon pathogenic challenges.

\section{Discussion}

The Drosophila SUMO-Targeted ubiquitin ligase Dgrn connects ubiquitylation and SUMOylation. Here, we report that Dgrn is required for both systemic and local responses to infection. dgrn heterozygous and null mutant flies are immuno-compromised and unable to express AMP genes upon infection, and rapidly succumbed to various pathogens. We found that Dgrn enhances the transcription of Toll and IMD target genes, and alleviated the repressive activity of the cytoplasmic inhibitor Cactus, as well as the nuclear co-repressor Gro. In addition, Dgrn is required within the adult mid-gut for the Notch-dependent stem cell regenerative response to infection, an activity that is counteracted by the ubiquitin-specific peptidases CG8334, which is highly similar to the mammalian USP11/USP32.

\section{Dgrn transcriptional activity}

We previously found that Dgrn functions as a "selector" that determines co-repressors recruitment during development (Abed et al., 2011). Our current study reveals that Dgrn activity is not limited to transcriptional repression, but that Dgrn is required for pathogenand signal-induced transcriptional activation of genes essential for innate immune response. However, while dgrn null mutants failed
A
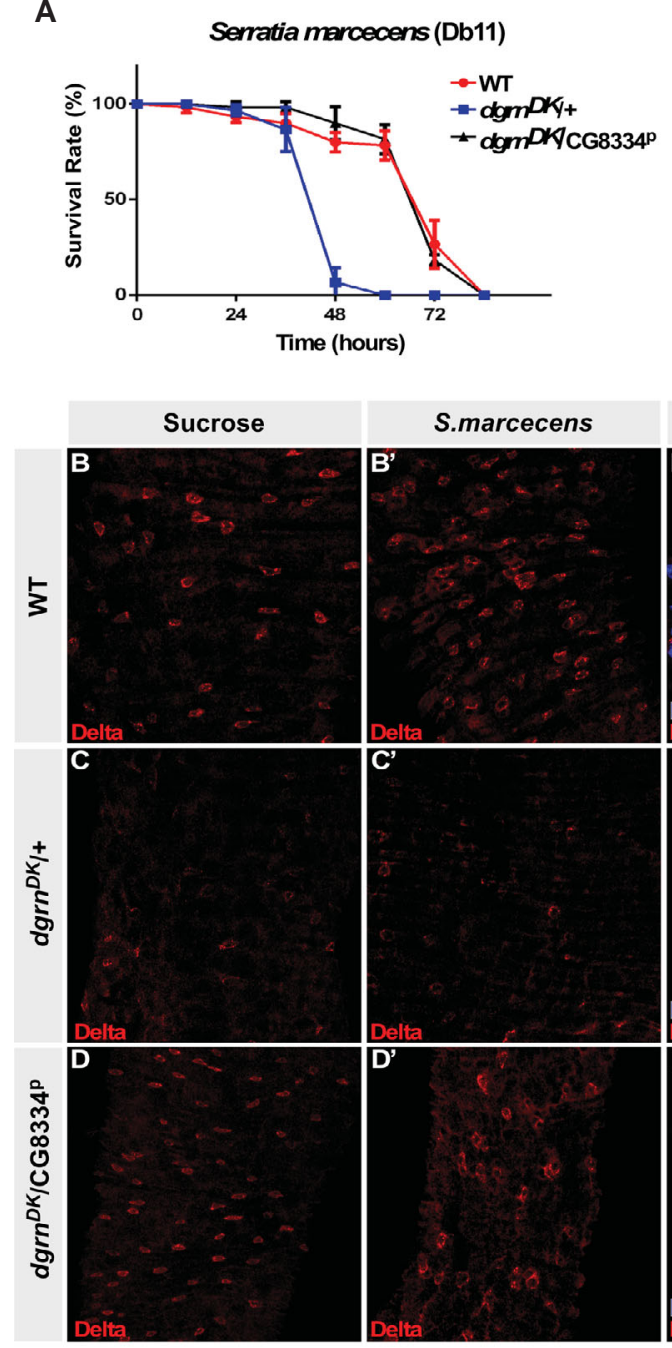

E

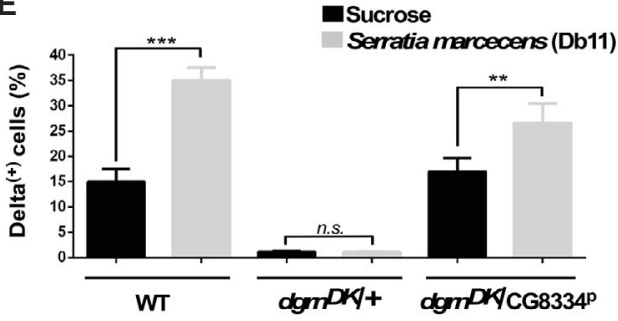

S.marcecens
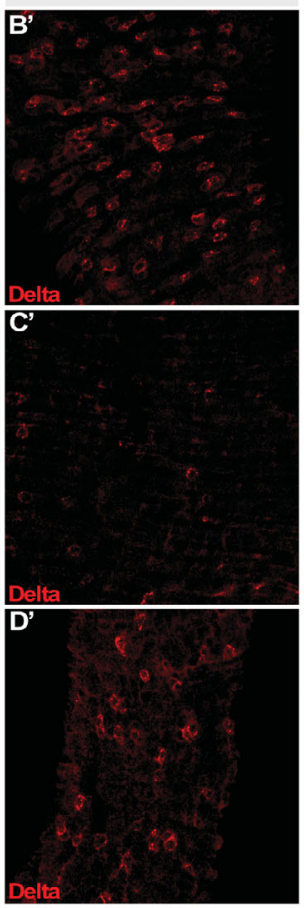

$A^{\prime}$
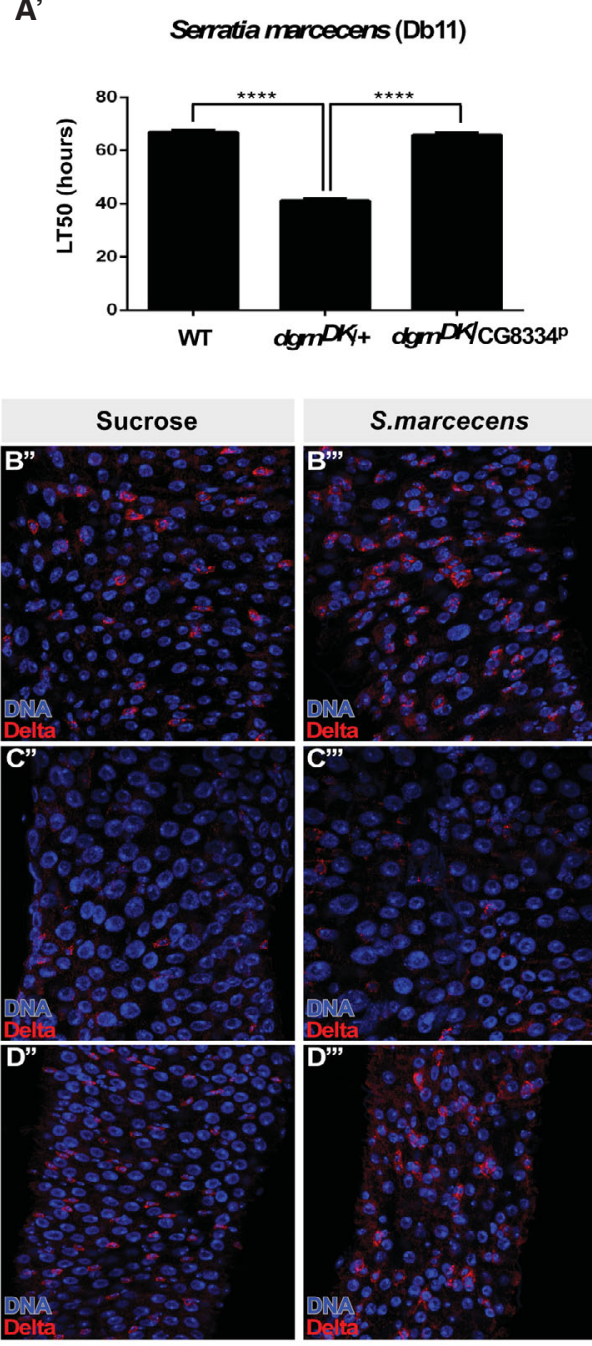

to express all endogenous AMPs tested, we found that dgrnnull mutants express established IMD- and Toll-reporter transgenes indifferently from control flies (data not shown). This difference suggests that the signaling pathways and basal transcriptional machinery are intact and are not the molecular target of Dgrn activity. Moreover, the inability to express endogenous AMP genes is in agreement with our previous report and unpublished data that during embryogenesis, dgrn null embryos fail to express mRNA of zygotic genes like twistand zenbut not maternal genes like nanos (Barry et al., Koltun, Orian and Paroush, personal communication). Taken together, we suggest that Dgrn activity may be related to a yet-to-be determined chromatin role of Dgrn, such as inhibiting the formation of local facultative heterochromatin, or the rapid opening of regulatory regions in

Fig. 5. The iso-peptidase CG8334 antagonizes dgrn. (A, $\left.\mathbf{A}^{\prime}\right)$ Survival of adult flies with the indicated genotypes upon infection with $\mathrm{Sm}$ (Db11). (A) Halving the dose of CG8334 (+ dgrnDK/CG8334 ${ }^{\mathrm{P}}+$ ) rescues the lethality observed in DgrnDK/+ heterozygous mutants. ( $\left.\mathbf{A}^{\prime}\right)$ LT50 is determined as the time in days when $50 \%$ of infected flies succumbed to infection. For pathogenic challenges $n=20$ for each genotype, and three independent biological repeats were performed in each set of experiments. (B-D'"'). Representative confocal images of Drosophila adult mid-gut of the indicated genotypes and $\alpha$-Delta antibody (RED) and DAPI (Blue) to visualized nuclei. Wild-type (B-B"'); dgrnDK/+ (C-C'"'); and + dgrn ${ }^{D K} / C G 8334^{P}$ $+\left(D-D^{\prime \prime \prime}\right)$. (B-D) Oral challenge with sucrose, $\left(B^{\prime}-D^{\prime}\right)$ Oral challenge with Sm. $B^{\prime \prime}-D^{\prime \prime}$ and $B^{\prime \prime \prime}-D^{\prime \prime \prime}$ are the merged images of $(B-D)$ and $\left(B^{\prime}-D^{\prime}\right)$, respectively, together with staining by DAPI (Blue) to visualized nuclei. In all experiments at least 7 individual guts were examined for each genotype, and three biological independent repeats were performed. (E) Quantification of \% Delta positive cells 48 h following oral infection with Sm(Db11) (gray bar) or sucrose (control, black bar). $n=250 P<0.01$. 
a "pioneer-like" manner (Liang et al., 2008; Harrison et al., 2011). This is in agreement with growing evidence of a role for chromatin remodelers and modifiers in the dampening of the innate immune response in Drosophila and in mammals (Bonnay et al., 2014, EMBO.J; Tartey el al., 2014, EMBO.J).

\section{The protein substrates of Dgrn}

It was previously shown that like Cactus, UBC9 and SUMOylation counteract immune response preventing inadequate activation and are required for limiting and terminating the immune response (Chiu et al., 2005; Paddibhatla et al., 2010). While the REL protein Dorsal is SUMOylated and this SUMOylation site is conserved in Dif and Relish (Bhashkar et al., 1997), our observations suggest Dif and Relish are not the targets for Dgrn activity. For example, the processing of Relish was intact in Dgrn-depleted S2 cells, and expression of Dgrn partially restored Toll-induced reporter activity in Dif-depleted cells. It is well established that in many cases, SUMOylation negatively regulates transcription, and many chromatin-related proteins are SUMOylated. Indeed, using Drosophila cells recent work identified proteins that undergo SUMOylation in response to LPS/Peptidoglycan challenge identifying nuclear proteins like HP1 that is involved in genes silencing (Handau et al., 2016). However, the identity of the proteins targeted by Dgrn during immune response is yet unknown, and we are pursuing an effort to identify these substrates.

\section{Dgrn and the local response to infection}

The regenerative response of the adult gut that is induced by infection is mediated by several signaling pathways such as Notch, JAK-STAT and EGFR (Jiang et al., 2012). We identified that dgrn is required for both basal and regenerative Notch signaling in the gut at the organismal level. However, we do not know the exact identity of the gut cell(s) where Dgrn is required, as expression of Dgrn in specific gut cells in the background of a dgrn null mutant was not sufficient for enabling the flies to survive infection or restore Delta expression (BK and $\mathrm{AO}$, personal communication). Thus, Dgrn may be required in multiple cells in the gut or for gut regeneration in cells outside the gut epithelia that have a critical impact on ISC. Yet, reducing the level of CG8334, an iso-peptidase whose human orthologs of USP11 is associated with RNF4 and antagonized RNF4 function, suppressed dgrn associated lethality and Delta expression, suggesting that wherever Dgrn is required for gut regeneration, its activity is antagonized by CG8334. Moreover, Dgrn likely regulates the activity of other pathways involved in regeneration in addition to Notch. For example, the small SUMO ligase PIAS1 (Protein inhibitory of JAK-STAT) negatively regulates JAK-STAT-dependent gene expression (Liu et al., 1998). Thus, targeting PIAS-SUMOylated substrates by Dgrn may relive the inhibitory activity of PIAS. Similarly, the transcription factors Yan and Pointed that mediate EGFR signaling were found to be SUMOylated (Handu et al., 2016) and are therefore potential nuclear substrates for Dgrn. Thus, Dgrn-dependentubiquitylation may serve as a molecular tool to relieve the inhibitory effect of SUMOylation on multiple substrates to enable gene activation.

To conclude, our study characterized the requirement for Dgrn in both systemic immune response and local regeneration upon infection in the gut. However, the exact mechanisms and specific substrates by which Dgrn acts during pathogen infection require further studies.

\section{Materials and Methods}

\section{Fly strains}

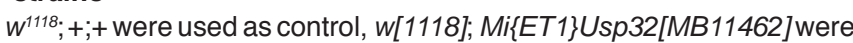
from the Bloomington stock center. $w^{1118 ;}+; d g r n^{D K}$ and UAS-Dgrn were as previously described (Berry et al., 2011). kenny ${ }^{\mathrm{co2831}}$ and myd88 ${ }^{\mathrm{c03881}}$ were described in (Tausszig-Delamasure et al., 2002), w; AttacinA::GFP;+, $P[w+m C$ Diptericin::LacZ, Drosomycin::GFP] were a kind gift of JL Imler (UPR9220, CNRS), the Notch reporter w;3.37 Gbe-Su(H)::Lac z;TM2/ TM6B was a kind gift of $S$. Bray. Flies were raised on standard cornmealyeast-agar medium at $25^{\circ} \mathrm{C}$ unless otherwise indicated.

\section{Plasmids}

pPac Drs-Luc, pTal-LacZ, pPGRP-LCa, pPac Toll ${ }^{\Delta L R R}$ were as previously described (GOTO et al., 2008). pNFKB-Luc-reporter was from Promega. $\mathrm{Cu}^{++}$-inducible pRMHA3 Dgrn, Dgrn ${ }^{\mathrm{CH} / \mathrm{AA}}$, Dgrn ${ }^{\mathrm{SIM}}$ and pMTVB Groucho were cloned $\mathrm{Kpn} / \mathrm{Xba}$ from the corresponding UAS-Dgrn and UAS-Gro vectors (EMBO) (Abed et al., 2011). pMTVB Cactus was cloned from the original Cactus cDNA bluescript vector, a kind gift of Ruth Steward. All plasmids were verified by sequencing.

\section{Primers}

Primers sets used for q-PCR (from applied biosystems):

$\begin{array}{lll}\text { Dm01822006_s1 } & \text { Drs } & \text { Drosomycin } \\ \text { Dm01818074_s1 } & \text { Def } & \text { Defensin } \\ \text { Dm01821460_s1 } & \text { Mtk } & \text { Metchnikowin } \\ \text { Dm02362218_s1 } & \text { AttA } & \text { Attacin-A } \\ \text { Dm02362226_s1 } & \text { AttB } & \text { Attacin-B } \\ \text { Dm01821391_g1 } & \text { AttC } & \text { Attacin-C } \\ \text { Dm02135981_g1 } & \text { AttD } & \text { Attacin-D } \\ \text { Dm01821557_g1 } & \text { DptA } & \text { Diptericin A } \\ \text { Dm01821449_s1 } & \text { Dro } & \text { Drosocin }\end{array}$

\section{Antibodies}

Mouse polyclonal $\alpha$-Dgrn (1:500, [Abed et al., 2011]); rabbit $\alpha$-Dif (1:5000, a kind gift from Tony IP); mouse $\alpha$-Actin (1:2000, MP Biomedicals 691001); Donkey $\alpha$-mouse IgG-HRP (1:10000; 715-035-150) and Goat $\alpha$-Rabbit HRP (1:10000, \#715-035-152) were from Jackson Laboratory. Mouse $\alpha$-Delta and $\alpha$-Prospero (1:50 and 1:100, respectively) were from DSHB. $\alpha$-Histon H3 (p-S10) 1:100 was from Abcam \#ab5176); Rabbit $\alpha$ $\beta$ Gal (1:500, MP Biomedicals \#55976), Alexa Fluor ${ }^{\circledR} 568$ goat $\alpha$-mouse IgG1 (1:1000, Invitrogen) and Alexa Fluor ${ }^{\circledR} 488$ goat $\alpha$-rabbit were from Invitrogen (1:1000, \#A21124 and \#A11008, respectively).

\section{Microbial strains, pathogenic challenge and exposure to Dextran Sulfate Sodium (DSS)}

Escherichia coli (1106) and Micrococcus luteus (CIPA270) bacteria for septic injuries were as described in (Reichhart et al., 2011, $\mathrm{n}=30$ ). Natural Beauveria bassiana infections were performed as previously described (Lemaitre et al., 1997). Serratia marcescens Db11 and Pseudomonas aeruginosa PA14 were used for oral infection (Bonnay et al., 2012).

\section{Oral exposure to Sm and DSS challenge}

Twenty 3- to 5-day-old flies of each genotype were assayed each time. Flies were transferred into an empty vial containing three absorbent $3 \mathrm{~mm}$ filters soaked with sucrose alone (control) or with sucrose and Sm bacteria. For DSS challenge, $400 \mu \mathrm{l}$ of $5 \%$ DSS in sucrose were used. Viability was counted daily, and surviving flies were transferred to new vials with fresh feeding media daily. For each pathogenic (pricking or oral challenges), or chemical challenge, at least three independent biological repeats were performed.

\section{S2 cell culture, transient transfections and RNAi targeting}

S2 were maintained in glutamine-enriched S2 Schneider media supplemented with $10 \%$ heat inactivated bovine calf serum. Transient transfections 
were performed using FuGENE-HD ${ }^{\circledast}$ (Promega), and RNAi was performed using MEGAscript RNAi Kit (Ambion AM1626) as previously described (Abed et al., 2011). The following specific primers were used:

GFP-i forward:

5'-gaattaatacgactcactatagggtgagcaagggcgaggagctg-3'

GFP-i reverse:

5'-gaattaatacgactcactatagggttgaagttcaccttgatgcc-3'

Dif-i forward:

5'- cggaattccgaattaatacgactcactatagggacaacaaactttgctaaatactagt-3'

Dif-i reverse:

5'- cggaaattccgaattaatacgactcactatagggaaattctaaagttatattttattta-3'

\section{Luciferase reporter assay}

Reporter assays in S2 Drosophila cells were performed similar to that described in (Abed et. al. 2011), with the following modifications: $3 \times 10^{6}$ cells were transfected where indicated with the following amounts of DNA: 100 ng pPac Drs-Lue, 100 ng pTAL NFkB-Lue, 100 ng pelican LacZ, 150 ng pPac PGRP-LCa, 50 ng pPac Toll $\Delta$ LRR (on Drs reporter) or $1000 \mathrm{ng}$ (on kB reporter), $300 \mathrm{ng}$ pRMHA3 vectors (Dgrn and its derived mutants), pMT/V5 Cactus, $150 \mathrm{ng}$ pMT/V5 Groucho, and pCDNA3 was used in order to make an equal amount of DNA. Eight hours post-transfection, $0.8 \mathrm{mM}$ $\mathrm{Cu}^{+2}$ was added, and 48 hours later, Luciferase activity was measured and normalized to $\beta$-galactosidase activity $(n=4)$.

\section{Western blot analysis}

Cell extracts were prepared using RIPA buffer, and $200 \mu$ g protein extract were resolved over SDS-PAGE and subjected to western blot analysis using the indicated antibodies similar to that described in (Abed et al., 2011).

\section{Determination of mRNA level of endogenous AMPs}

RNA extraction was performed using TRI reagent (T9424 Sigma). Total RNA was converted to cDNA using qScript cDNA Synthesis kit (95047-100, Quanta Bioscience) according to manufacturer's instructions. Expression levels of each Amp gene were detected using TaqMan ${ }^{\circledR}$ with the indicated primers. qPCR was performed according to manufacturer's instructions. All samples were analyzed in triplicate, and the levels of detected mRNA were normalized to control Rp49 mRNA values. Normalized data were used to quantify the relative levels of a given mRNA according to cycling threshold analysis. Target gene expression in the uninfected flies was set arbitrarily as 1 , and results were presented as relative expression levels.

\section{Dissection of Drosophila gut and indirect immunofluorescence}

Gut dissection and immunofluorescence detection: gut fixation and staining was carried out similar to that previously described (SHAW et. al. 2010). Guts were then incubated overnight at $4^{\circ} \mathrm{C}$ with $100 \mu \mathrm{l}$ first antibody diluted in blocking buffer (for immunostaining) with gentle rocking. The following day, guts were washed $\mathrm{x} 4$ in PTX (15 min per wash with gentle rocking) at room temperature and then incubated for $1 \mathrm{~h}$ with 100 $\mu$ fluorescent secondary antibody $(1: 1000)$ diluted in blocking buffer (for immunostaining). DAPI (Sigma D9542, 1:1000 of a $1 \mu \mathrm{g} / \mu \mathrm{l}$ stock solution) was added to the secondary antibody solution and covered with foil. Guts were washed with PTX-x2 (15\%/wash), and an additional time with PBSx1 for $15 \mathrm{~min}$. Subsequently, guts were mounted onto a microscope slide with two drops of mounting solution (Fluoromount, Southern Biotech, 0100-01) and analyzed using the Zeiss LSM 710 META laser scanning confocal microscope.

\section{Statistical analysis}

Reporter assays were analyzed statistically using SEM and t-test, and comparisons were performed using the GraphPad Prism 5.00 (GraphPad Software, San Diego, CA, USA). Significance is indicated by ${ }^{* *}=P<0.001$ and ${ }^{* *}=P<0.01 . n=5$.

LT50 (lethal time in days at which $50 \%$ of the flies died) analysis was calculated using the GraphPad Prism 5.00 (GraphPad Software, San Diego, CA, USA). Log-rank (Mantel-Cox) test was performed. Survival experiments shown are representative of at least three independent experiments where each 25 individuals were tested for each genotype.

\section{Acknowledgments}

We thank members of the Orian lab for their comments and assistance. We are grateful to Z. Paroush, D. Chamovich, B. Edgar, S. Bray and JL Imler for sharing their antibodies and fly lines. This research was published under the frame work of the LABEX:ANR-10-LABX-0036_NETRNA, and funding of the state managed by the French National Research Agency as part of the Investments for the future program to NM and JMR. The Orian lab was supported by ISF \#739-2015, the Goldy Family Research Fund, and Rappaport Research Fund.

\section{References}

ANDERSON K V, JURGENS G, NUSSLEIN-VOLHARD C (1985). Establishment of dorsal-ventral polarity in the Drosophila embryo: genetic studies on the role of the Toll gene product. Cell 42: 779-789.

ABED M, BARRY K C, KENYAGIN D, KOLTUN B, PHIPPEN T M, DELROW J J, PARKHURST S M, ORIAN A (2011). Degringolade, a SUMO-targeted ubiquitin ligase, inhibits Hairy/Groucho-mediated repression. EMBO J 30: 1289-1301.

BARRYKC, ABED M, KENYAGIND, WERWIETR, BOICOO, ORIANA, PARKHUTST S M (2011). The Drosophila STUbL protein Degringolade limits HES functions during embryogenesis. Development 138: 1759-1769.

BONNAY F, NGUYEN X H, COHEN-BERROS E, TROXLER L, BATSCHE E, CAMONIS J, TAKEUCHI O, REICHHART J M, MATT N. (2014) Akirin specifies NF-KB selectivity of Drosophila innate immune response via chromatin remodeling. EMBO J. 33: 2349-2362.

BUCHON N, SILVERMAN N, CHERRY S (2014). Immunity in Drosophila melanogaster - from microbial recognition to whole-organism physiology. Nat Rev Immunol 14: 796-810.

CINNAMON E, PAROUSH Z (2008). Context-dependent regulation of Groucho/TLEmediated repression. Curr Opin Genet Dev 18: 435-440.

CHIU H, RING B C, SORRENTINO R P, KALAMATZM, GARZA D, GOVIND S (2005). dUbc9 negatively regulates the Toll-NF-kappa $B$ pathways in larval hematopoiesis and drosomycin activation in Drosophila. Dev Biol 288: 60-72.

DECQUE A, JOFFERE O, MAGALHAES J G, COSSEC J C, BLECHER-GONEN R, LAPAQUETTE P, SILVIN A, MANEL N, JOUUBERT P E, DEJEAN A. et al., (2016). Sumoylation coordinates the repression of inflammatory and anti-viral gene-expression proGrams during innate sensing. Nat Immunol 17: 140-149.

EVERRET R D, BOUTELL C, HALE B G (2013). Interplay between viruses and host sumoylation pathways. Nat Rev Microbiol 11: 400-411.

FERRNADON D, IMLER J L, HETRU C, HOFFMANN J A. (2007). The Drosophila systemic immune response: sensing and signalling during bacterial and fungal infections. Nat Rev Immunol. 7: 862-874.

FERRANDON D. (2013) The complementary facets of epithelial host defenses in the genetic model organism Drosophila melanogaster: from resistance to resilience. Curr Opin Immunol. 1: 59-70.

FLTHO A, MELCHIOR F. (2013). Sumoylation: a regulatory protein modification in health and disease. Annu Rev Biochem 82: 357-385.

GHOSH S, HAYDEN M S (2012). Celebrating 25 years of NF-kB research. Immunol Rev 246: 5-13.

GOTO A, MATSUSHITA K, GESELLCHEN V, EI CHAMY L, KUTTENKEULER D, TAKEUCHI O, HOFFMANN J A, AKITAS, BOUTROS M, REICHAART J M. (2008) Akirins are highly conserved nuclear proteins required for NF-kappaB-dependent gene expression in Drosophila and mice. Nat Immunol 9: 97-104.

HEATON S M, BORG N A, DIXIT V M (2016). Ubiquitin in the activation and attenuation of innate antiviral immunity. $J$ Exp Med 213: 1-13.

HANDU M, KADUSKAR B, RAVINDRANATHAN R, SOORYA, GIRI R, ELANGO V B, GOWDAH, RATNAPARKHI G S (2015). SUMO-enriched proteome for Drosophila innate immune response. G3 5: 2137-2154.

HARRISON M M, LI X Y, KAPLAN T, BOTCHAN M R, EISEN M B (2011). Zelda binding in the early Drosophila melanogasterembryo marks regions subsequently activated at the maternal-to-zygotic transition. PLoS Genet 7:e1002266.

HENDRIKS I A, SCHIMMEL J, EIFLER K, OLSEN J V, VERTEGAAL A C (2015). 
Ubiquitin-specific protease 11 (USP11) deubiquitinates hybrid small ubiquitin-like modifier (SUMO)-ubiquitin chains to counteract RING finger protein 4 (RNF4). J Biol Chem 290: 15526-15537.

HERIDE C, URBE S, CLAGUE M J (2014). Ubiquitin code assembly and disassembly. Curr Biol 24: R215-220.

JIANG H, EDGAR B A (2012). Intestinal stem cell function in Drosophila and mice. Curr Opin Genet Dev. 22: 354-360.

KANAREK N, BEN-NERIAH Y (2012). Regulation of NF-kB by ubiquitination and degradation of the IKBs. Immunol Rev 246: 77-94.

LEE J H, PARK S M, KIMOS, LEE C S, WOO J H, PARK S J, JOE E H, JOU I (2009). Differential SUMOylation of LXRalpha and LXRbeta mediates trans-repression of STAT1 inflammatory signaling in IFN-gamma-stimulated brain astrocytes. $\mathrm{Mol}$ Cell 35: 806-817.

LEE M H, MABB A M, GILL G B, YEH E T, MIYAMOTO S (2011). NFKB induction of the SUMO protease SENP2: a negative feedback loop to attenuate cell survival response to genotoxic stress. Mol Cell 43: 180-91.

LEMAITRE B, HOFFMANN J (2007). The host defense of Drosophila melanogaster. Annu Rev Immunol 2007 25: 697-743.

LIANG H L, NIEN C Y, LIU H Y, METZSTEIN M M, KIROV N, RUSHLOW C (2008). The zinc-finger protein Zelda is a key activator of the early zygotic genome in Drosophila. Nature 456: 400-403.

LIU B, LIAO J, RAO X, KUSHNER S A, CHUNG C D, CHANG D D, SHUAI K (1998). Inhibition of Stat1-mediated gene activation by PIAS1. Proc Natl Acad Sci USA. 95: 10626-10631.

MENG X, KHANUJA B S, IP Y T. (1999) Toll receptor-mediated Drosophila immune response requires Dif, an NF-kB factor. Genes Dev 13: 792-797.

PADDIBHATLA I, LEE M J, KALAMRTZ M E, FERRERESE R, GOVIND S (2010). Role for SUMOylation in systemic inflammation and immune homeostasis in Drosophila larvae. PLoS Pathog. 6:e1001234.
POPOVIC D, VUCIC D, DICKIC I (2014). Ubiquitination in disease pathogenesis and treatment. Nat Med 20: 1242-1253.

RIBET D, COSSART P (2010). Listeria monocytogenes impairs SUMOylation for efficient infection. Nature 464: 1192-1195.

RYU T, SPATOLA B, DELEBARE L, BOWLIN K, HOPP H, KUNITAKE R, KARPEN G H, CHIOLO I (2015). Heterochromatic breaks move to the nuclear periphery to continue recombinational repair. Nat Cell Biol. 17: 1401-1411.

SHAW R L, KOHLMAIER A, POLESELLO C, VEELKEN C, EDGAR B A, TAPON $N$ (2010). The Hippo pathway regulates intestinal stem cell proliferation during Drosophila adult midgut regeneration. Development 137: 4147-4158.

SRIRAMACHANDRANA M, DOHMEN R J (2014). SUMO-targeted ubiquitin ligases. Biochim Biophys Acta 21843: 75-85.

TARTEY S, MATSUSHITAK, VANDENBONA, ORI D, IMAMURAT, MINOT, STANDLEY D M, HOFFMANN J A, RERICHHHART J M, AKIRAS, TAKEUUCHIO.(2014) Akirin2 is critical for inducing inflammatory genes by bridging IKB- $\zeta$ and the $\mathrm{SWI} /$ SNF complex. EMBO J. 33: 2332-4238.

TAUSZIG-DELAMASURE S, BILAK H, CAPOVILLA M, HOFFMANN J A, IMLER J L. (2002) Drosophila MyD88 is required for the response to fungal and Gram-positive bacterial infections. Nat Immunol 2002 3: 91-97.

WAN F, LENARDO M J. (2010). The nuclear signaling of NF-кB: current knowledge, new insights, and future perspectives. Cell Res 20: 24-33.

WI H C, LIN Y C, LIU C H, CHUNG H C, WANG Y T, LIN Y W, MAHI, TU PH, LAWLER S E, CHEN R H (2014). USP11 regulates PML stability to control Notch-induced malignancy in brain tumours. Nat Commun 5: 3214.

YU M, LIU K, MAO Z, LUO J, GU W, ZHAO W (2016). USP11 is a Negative Regulator to $\gamma \mathrm{H} 2 \mathrm{AX}$ Ubiquitylation by RNF8/RNF168. J Biol Chem 291: 959-967.

van der VEEN A G, PLEOGH H L (2012). Ubiquitin-like proteins. Annu Rev Biochem 81: 323-357. 


\section{Further Related Reading, published previously in the Int. J. Dev. Biol.}

Ontogeny of the Drosophila larval hematopoietic organ, hemocyte homeostasis and the dedicated cellular immune response to parasitism

Joanna Krzemien, Michele Crozatier and Alain Vincent

Int. J. Dev. Biol. (2010) 54: 1117-1125

http://dx.doi.org/10.1387/ijdb.093053jk

The deubiquitylating enzyme Cops6 regulates different developmental processes during early zebrafish embryogenesis William K.F. Tse, May-Su You, Steven Hao-Kee Ho and Yun-Jin Jiang Int. J. Dev. Biol. (2011) 55: 19-24

http://dx.doi.org/10.1387/ijdb.103089wt

Mammalian hibernation: differential gene expression and novel application of epigenetic controls

Pier Morin Jr. and Kenneth B. Storey

Int. J. Dev. Biol. (2009) 53: 433-442

http://dx.doi.org/10.1387/ijdb.082643pm

Role of polycomb proteins Ring1A and Ring1Bin the epigenetic regulation of gene expression

Miguel Vidal

Int. J. Dev. Biol. (2009) 53: 355-370

http://dx.doi.org/10.1387/ijdb.082690mv

Regulation of cell fate determination by Skp1-Cullin1-F-box (SCF) E3 ubiquitin ligases Christopher J. Hindley, Gary S. McDowell, Helen Wise and Anna Philpott Int. J. Dev. Biol. (2011) 55: 249-260

http://dx.doi.org/10.1387/ijdb.103171ch

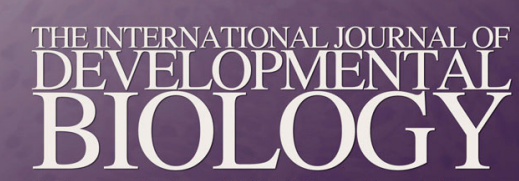

Volume 60 Nos. 10/11/12

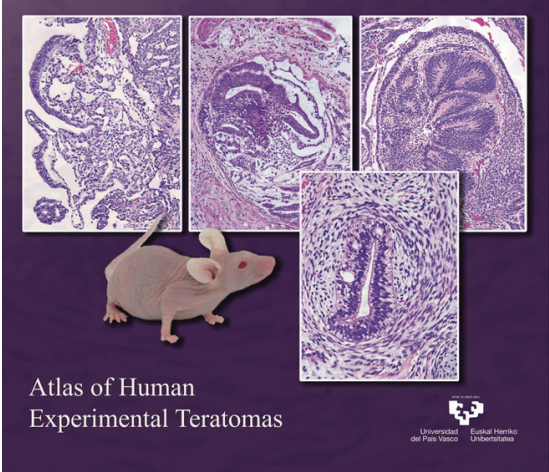

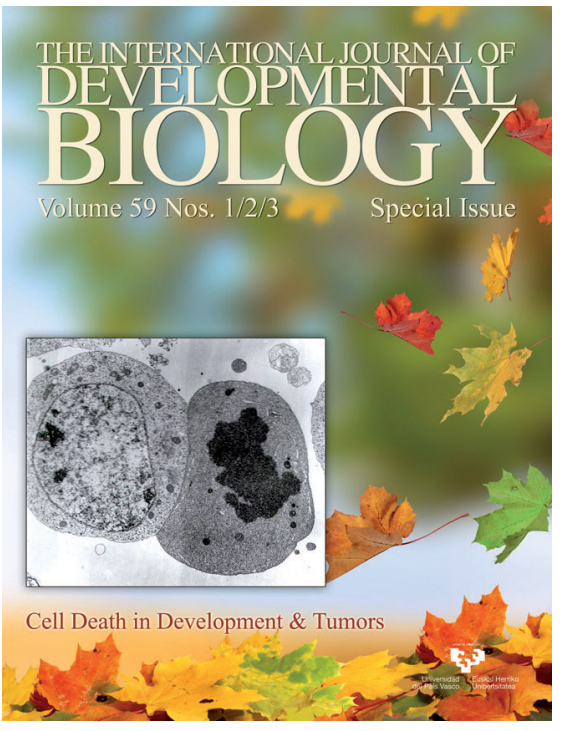
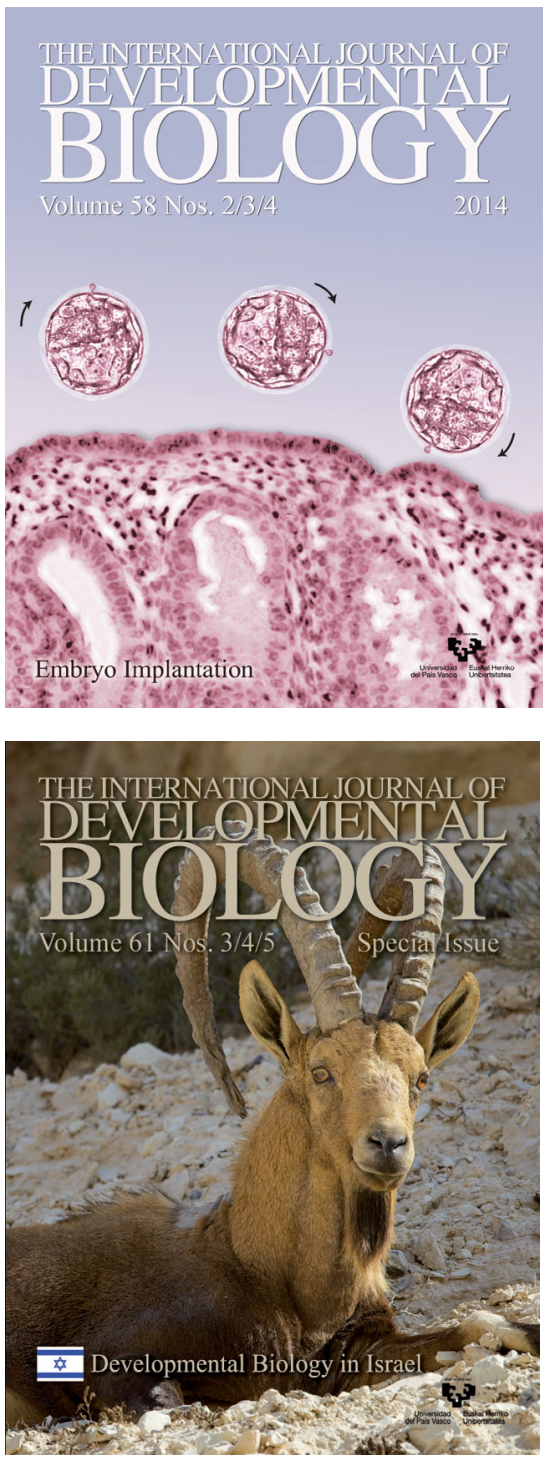